\title{
Legionella drancourtii sp. nov., a strictly intracellular amoebal pathogen
}

\author{
Correspondence \\ Didier Raoult \\ raoult@medecine.univ-mrs.fr
}

\author{
Bernard La Scola, ${ }^{1}$ Richard J. Birtles, ${ }^{2}$ Gilbert Greub, ${ }^{1}$ \\ Timothy J. Harrison, ${ }^{3}$ Rodney M. Ratcliff ${ }^{4}$ and Didier Raoult ${ }^{1}$ \\ ${ }^{1}$ Unité des Rickettsies, CNRS UPRESA 6020, Faculté de Médecine, Université de la \\ Méditerrannée, 27 Boulevard Jean Moulin, 13385 Marseille Cedex 05, France \\ 2Department of Veterinary Pathology, Faculty of Veterinary Science, University of Liverpool,
Leahurst, Neston CH64 7TE, UK \\ ${ }^{3}$ Respiratory and Systemic Infections Laboratory, Central Public Health Laboratory, \\ Public Health Laboratory Service, London NW9 5HT, UK \\ ${ }^{4}$ Infectious Diseases Laboratories, Institute of Medical and Veterinary Science, Frome Road, \\ Adelaide, SA 5000, Australia
}

\begin{abstract}
A Legionella-like amoebal pathogen (LLAP), formerly named LLAP12 ${ }^{\top}$, was characterized on the basis of microscopic appearance, staining characteristics, growth in Acanthamoeba polyphaga at different temperatures, DNA G $+C$ content, serological cross-reactivity and 16S rRNA and macrophage infectivity potentiator ( $\mathrm{mip}$ ) gene sequence analysis. LLAP $12^{\top}$ was found to be a motile, Gram-negative bacterium that grew within cytoplasmic vacuoles in infected amoebae. The infecting bacteria induced lysis of their amoebal hosts and time taken to do so was dependent on incubation temperature. Recovery of LLAP $12^{\top}$ from amoebae onto axenic media could not be achieved. Phylogenetic analysis of LLAP12 ${ }^{\top}$, based on $16 \mathrm{~S}$ rRNA and mip gene sequence analysis, indicated that it lay within the radiation of the Legionellaceae and that it clustered specifically with Legionella lytica and Legionella rowbothamii. The divergence observed between LLAP $12^{\top}$ and these two species was of a degree equal to, or greater than, that observed between other members of the family. In support of this delineation, LLAP1 ${ }^{\top}$ was found not to cross-react serologically with any other Legionella species. The mip and $16 \mathrm{~S}$ rRNA gene sequence-based analyses also indicated that $L L A P 12^{\top}$ was related very closely to two other previously identified LLAP isolates, LLAP4 and LLAP11. Taken together, these results support the proposal of LLAP12 ${ }^{\top}$ as the type strain of Legionella drancourtii sp. nov.
\end{abstract}

Intracellular parasitism of protozoa by bacteria is an increasingly recognized phenomenon. Adaptation to this niche has occurred independently among different groups of bacteria: extant intra-protozoal parasites are phylogenetically diverse. Five lineages have been identified among the obligate endosymbionts (Horn et al., 2001) and this diversity is matched by that of facultative intraamoebal parasites, which include numerous established human pathogens (Barker \& Brown, 1994; Horn et al., 2001). Following the demonstration that legionellae exploit amoebae as natural hosts (Rowbotham, 1980, 1983), amoebal co-culture methods have been introduced for the recovery of legionellae from environmental samples

Abbreviations: LLAP, Legionella-like amoebal pathogen; mip, macrophage infectivity potentiator.

The GenBank/EMBL/DDBJ accession number for the 16S rRNA gene sequence of $L$. drancourtii LLAP12 ${ }^{\top}$ is $X 97366$ and that for the mip gene sequence is AF407571. and have led to the isolation of several organisms that could not be cultured on axenic media (Rowbotham, 1983; Barbaree et al., 1986). As these organisms could not be subjected to conventional methods of bacterial identification, their designation remained Legionella-like amoebal pathogens (LLAPs). The introduction of 16S rRNA gene PCR-based methods finally permitted partial characterization of LLAPs and, on the basis of 16S rRNA gene sequence comparison, many were found to share specific evolutionary homology with members of the Legionellaceae (Fry et al., 1991; Birtles et al., 1996). Although several LLAPs have now been grown axenically (Adeleke et al., 2001), cultivation of others has failed. In this study, an LLAP isolate that had previously been characterized only by $16 \mathrm{~S}$ rRNA gene sequence analysis (Birtles et al., 1996) was subjected to polyphasic assessment, thereby permitting its accurate classification.

LLAP $12^{\mathrm{T}}$ was isolated by T. J. Rowbotham (Public Health Laboratory, Leeds, UK) in co-culture with an Acanthamoeba 
species from an environmental water source in West Yorkshire, UK (Fallon \& Rowbotham, 1990). In our laboratory, LLAP $12^{\mathrm{T}}$ was co-cultured routinely with Acanthamoeba polyphaga strain Linc AP-1 in peptone/yeast extract/ glucose medium under previously described conditions at $32{ }^{\circ} \mathrm{C}$ (Rowbotham, 1983). All attempts to isolate LLAP $12^{\mathrm{T}}$ by using buffered charcoal/yeast extract agar failed, despite the use of media from different manufacturers (bioMérieux, Diagnostic Pasteur) and prolonged incubation at 30 or $35^{\circ} \mathrm{C}$, with or without the presence of $5 \% \mathrm{CO}_{2}$.

\section{Phenotypic characterization}

The microscopic appearance of LLAP12 ${ }^{\mathrm{T}}$ was verified by direct observation of infected amoebae using an inverted microscope, and of fixed and stained bacteria by using conventional microscopy. Both approaches revealed large numbers of motile bacteria within amoebal cytoplasmic vacuoles. These organisms stained weakly with Gram stain (negative), but well with Giménez stain. Ultrastructural analysis using transmission electron microscopy (La Scola et al., 2001) indicated that LLAP $12^{\mathrm{T}}$ was a polymorphic, Gram-negative organism and confirmed that clusters of bacteria lay within amoebal cytoplasmic vacuoles (Fig. 1).

Purification of $\mathrm{LLAP}^{\mathrm{T}}{ }^{\mathrm{T}}$ (for inoculation into animals) and serology were performed as described previously (La Scola et al., 2001), as was the production of polyvalent mouse antibodies (La Scola et al., 2001). This antiserum

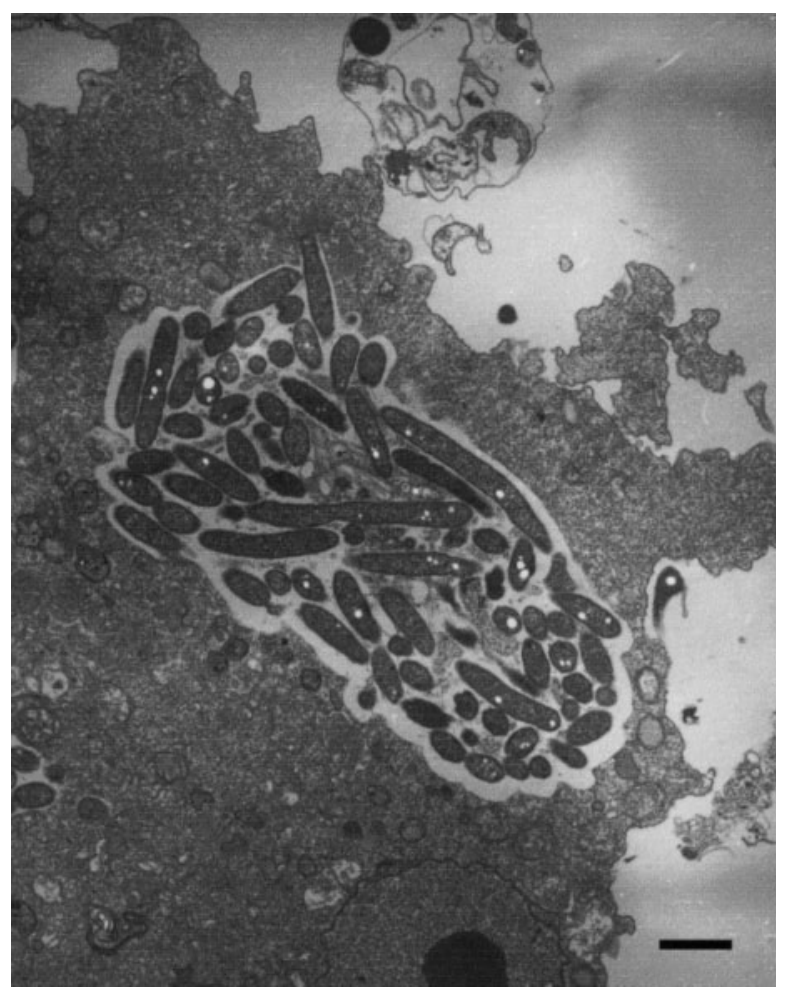

Fig. 1. Transmission electronic micrograph of $L L A P 12^{\top}$ within vacuoles of $A$. polyphaga. Bar, $1 \mu \mathrm{m}$. was adsorbed to the amoebae by mixing the sera with washed amoebae, then shaking the mixture overnight at room temperature. After centrifugation at $10000 \mathrm{~g}$ for $10 \mathrm{~min}$, the supernatant was removed and frozen at $-80^{\circ} \mathrm{C}$. The immunofluorescent test (Harrison \& Taylor, 1988) was used to assess cross-reactivity between antisera that were raised against LLAP12 ${ }^{\mathrm{T}}$ and 69 different Legionella antigens, and cross-reactivity between LLAP $12^{\mathrm{T}}$ and rabbit antisera that were raised against 80 Legionella antigens. Despite a high $(>1 / 3200)$ homologous antibody titre to LLAP12 ${ }^{\mathrm{T}}$, the mouse antiserum failed to react significantly $(>1 / 100)$ with any other Legionella antigen preparation. Furthermore, all antisera that were raised against other Legionella taxa failed to react significantly $(>1 / 100)$ with LLAP $12^{\mathrm{T}}$ antigen.

Our observations of LLAP $12^{\mathrm{T}}$ morphology and its intraamoebal position are in keeping with those reported for other legionellae. Although serological reactivity among members of the Legionellaceae varies, this approach has long served as a routine method for species identification. It is reasonable to suspect that an isolate that demonstrates no reactivity with a panel of antisera that represents the serological spectrum of the current genus is likely to be a member of a novel taxon.

\section{Sequence analysis}

Methods used for amplification and determination of $16 \mathrm{~S}$ rRNA and macrophage infectivity potentiator (mip) gene sequences and the procedure for determination of DNA $\mathrm{G}+\mathrm{C}$ content have been detailed elsewhere (Birtles et al., 1996; Ratcliff et al., 1998; La Scola et al., 2001). A partial mip gene sequence for Legionella taurinenesis Turin $\mathrm{I}^{\mathrm{T}}$ was also determined. A $585 \mathrm{bp}$ fragment of the mip gene of LLAP $12^{\mathrm{T}}$ was sequenced and used for further analysis. Sequence comparison revealed that the LLAP $12^{\mathrm{T}}$ sequence was most similar (86-88 \%) to those of Legionella lytica and Legionella rowbothamii. Phylogenetic reconstruction, derived from an alignment of the LLAP $12^{\mathrm{T}}$ mip gene sequence fragment with those of Legionella species with validly published names by using previously described methods (Birtles et al., 1996; Ratcliff et al., 1998), revealed that LLAP12 ${ }^{\mathrm{T}}$ clustered specifically with, but had diverged from, L. lytica and $L$. rowbothamii (Fig. 2). This branching order was supported strongly by bootstrap analysis. Interestingly, the LLAP $12^{\mathrm{T}}$ mip gene sequence was found to be indistinguishable from that of LLAP4, an organism that was isolated in the same manner as LLAP $12^{\mathrm{T}}$ and was characterized partially by $16 \mathrm{~S}$ rRNA gene sequence analysis (Birtles et al., 1996). Although this earlier study found that among the Legionellaceae, LLAP4 was related specifically to LLAP $12^{\mathrm{T}}$, there was, nonetheless, $1 \cdot 3 \% 16 \mathrm{~S}$ rRNA gene sequence dissimilarity between them (19 mismatches in an alignment of $1482 \mathrm{bp}$ ). This was the first time that interstrain $16 \mathrm{~S}$ rRNA gene sequence dissimilarity had been found to be greater than that observed between mip gene sequences. Furthermore, the extent of $16 \mathrm{~S}$ rRNA gene sequence dissimilarity between LLAP4 and LLAP $12^{\mathrm{T}}$ was greater than that seen between some different Legionella 

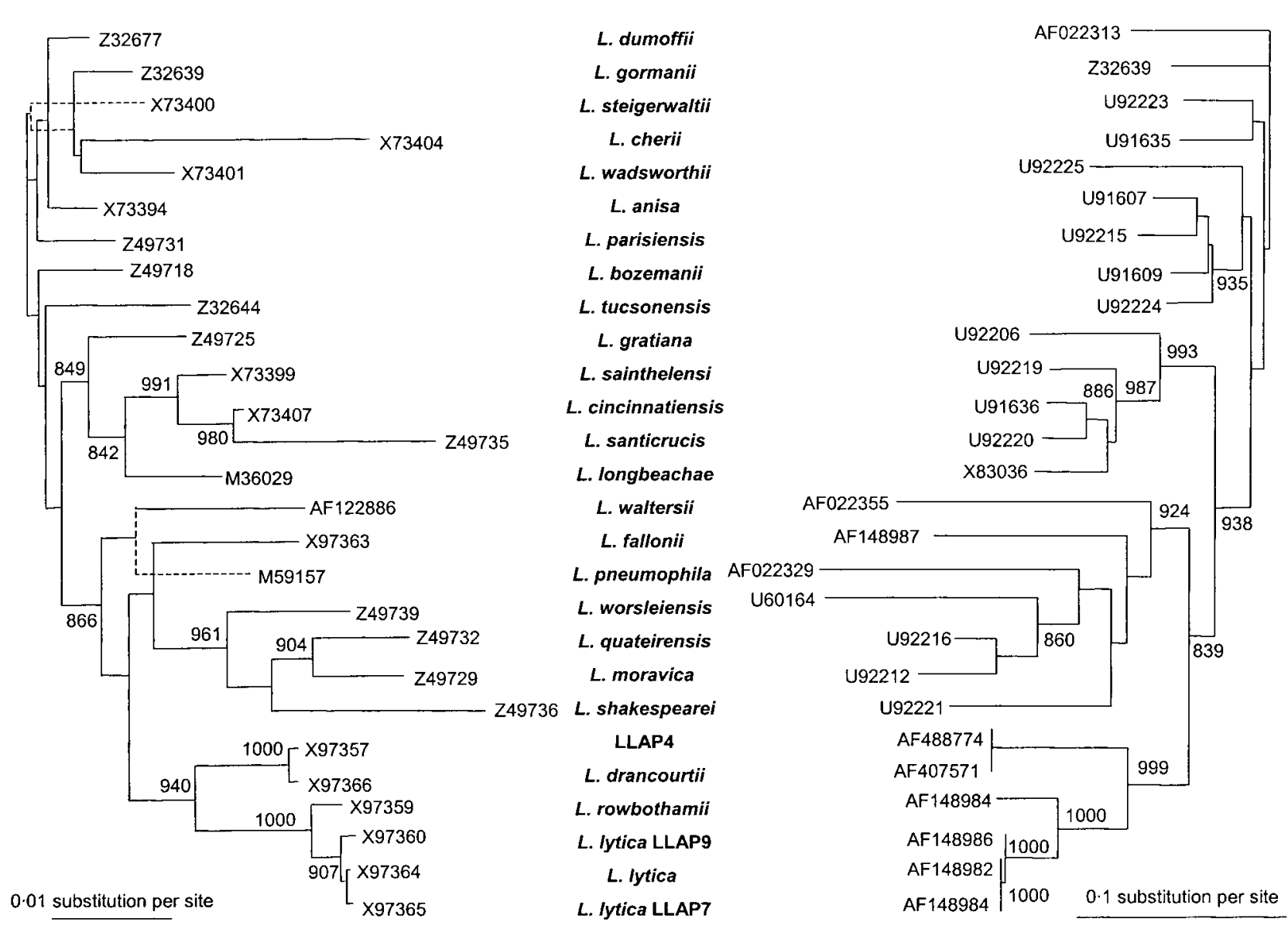

Fig. 2. Dendrogram representing phylogenetic relationships between $L$. drancourtii $\mathrm{LLAP}_{1} 2^{\top}$ and other closely related members of the genus Legionella. Dendrograms were generated from mip gene fragments (right-hand tree) and the 16S rRNA gene (left-hand tree) by using the neighbour-joining method. Support of each branch, as determined from 1000 bootstrap resamples, is indicated by the value at the node when $\geqslant 750$.

species (the 16S rRNA genes of $L$. rowbothamii and L. lytica differ by $<1 \%$ ). Concern about these observations led us to repeat 16S rRNA gene sequencing for LLAP4 and LLAP12 $^{\mathrm{T}}$. Although LLAP4 was no longer extant, DNA preparations remained. The $16 \mathrm{~S}$ rRNA gene sequences we obtained from LLAP $12^{\mathrm{T}}$ and LLAP4 were different from those submitted to GenBank (accession numbers X97366 and X97357, respectively). The new LLAP12 ${ }^{\mathrm{T}}$ sequence differed at only two positions from that submitted to GenBank (in a 1348 bp alignment), whereas the new LLAP4 sequence differed at 12 positions (in a $1350 \mathrm{bp}$ alignment). Comparison of the new LLAP4 and LLAP $12^{\mathrm{T}} 16 \mathrm{~S}$ rRNA gene sequences indicated that they differed at only four sites $(<0 \cdot 3 \%$ of a 1348 bp alignment). Our experiences serve as a warning that sequences held in databases, particularly those deposited in the 1980s and early 1990s when sequencing methodologies were somewhat cruder than those that are currently available, cannot always be relied on. The LLAP4 and LLAP $12^{\mathrm{T}}$ 16S rRNA gene sequences held by GenBank have now been revised.
Comparison of $16 \mathrm{~S}$ rDNA sequences reveals that LLAP12 ${ }^{\mathrm{T}}$ shares $97 \%$ similarity with L. lytica $\mathrm{L}^{\mathrm{T}}$ and L. rowbothamii LLAP-6 ${ }^{\mathrm{T}}$ and $98 \%$ similarity with L. lytica LLAP7. As it has been demonstrated previously that organisms sharing $>97 \%$ similarity may belong to the same species (Ash et al., 1991; Fox et al., 1992; Stackebrandt \& Goebel, 1994), this analysis should not be relied on in the justification of species status for LLAP12 ${ }^{\mathrm{T}}$. However, the 16S rRNA gene sequences of other Legionella species are more similar to one another than that of LLAP $12^{\mathrm{T}}$ is to that of L. lytica. Furthermore, phylogenetic inferral derived from 16S rRNA gene sequence data demonstrates clearly that LLAP $12^{\mathrm{T}}$ has diverged significantly from its close relatives. A previous study using these data (Birtles et al., 1996) included LLAP4 and LLAP11, organisms that are no longer viable and thus could not be included in the current study. LLAP11 was found to possess a $16 \mathrm{~S}$ rRNA gene sequence that was indistinguishable from that of LLAP $12^{\mathrm{T}}$, whereas LLAP4 was shown in this study to possess a sequence that shares $>99 \%$ similarity with that of LLAP $12^{\mathrm{T}}$. Taken together, 
these data provide further evidence that $\operatorname{LLAP}^{\mathrm{T}} 2^{\mathrm{T}}$ is not unique, but rather represents a distinct clade of legionellae.

Phylogenetic analyses derived from 16S rRNA and mip gene analysis are generally congruent (Ratcliff et al., 1998), although with variation within the genus Legionella of up to $30 \%$, mip gene sequences potentially offer a more reliable and sensitive basis for comparative analysis. Nonetheless, the combined taxonomic value of 16S rRNA and mip gene sequence analyses is greater than the sum of their individual worth, as congruence in bi- or multilocus analyses provides a good indication that evolutionary relationships inferred from individual genes or gene fragments do indeed represent true phylogenies for the organism. Thus, these data and those derived from the serological assessment provide unequivocal evidence that LLAP $12^{\mathrm{T}}$ is a representative of a novel Legionella species. The data also support the inclusion of LLAP4 and LLAP11 in this species, a hypothesis that was first put forward solely on the basis of 16S rRNA gene sequence data (Adeleke et al., 1996).

\section{Effect of temperature on the physiology of intra-amoebal LLAP12 ${ }^{\top}$}

To assess the effect of temperature on the nature of the association between LLAP $12^{\mathrm{T}}$ and its amoebal host, six flasks that each contained $10 \mathrm{ml}$ of a suspension of washed amoebae $\left(5 \times 10^{5} \mathrm{ml}^{-1}\right)$ in Page's amoeba saline (PAS) solution were seeded with $1 \mathrm{ml}$ supernatant from a 7-dayold infected flask to yield a bacteria: cell ratio of $10: 1$. Infected flasks were incubated at $25,28,30,32,35$ and $37^{\circ} \mathrm{C}$. Every day for 8 days, flasks were shaken, then $100 \mu \mathrm{l}$ culture was removed, mixed with $300 \mu \mathrm{l}$ trypan blue $(0.13 \%)$ (Sigma-Aldrich) in PAS solution, then transferred to a Nageotte counting chamber. Microscopic examination of amoebae permitted the enumeration of intact cells. Six uninfected amoebal cultures that were prepared concurrently with those described above and incubated and handled in the same way were used as controls.

A. polyphaga grew at all temperatures tested, but higher numbers of cells survived for longer periods at lower incubation temperatures (Fig. 3). Conversely, when infected with LLAP $12^{\mathrm{T}}$, amoebae incubated at these lower temperatures $\left(<35^{\circ} \mathrm{C}\right)$ lysed more rapidly (Fig. 3). After $48 \mathrm{~h}$, LLAP $12^{\mathrm{T}}$ infection led to lysis of $53 \%$ of amoebae at $25-32{ }^{\circ} \mathrm{C}$, as opposed to $0 \%$ at $35-37^{\circ} \mathrm{C}(P<0 \cdot 05)$. This observation was confirmed by phase-contrast microscopic observation of infected amoebal cultures in culture flasks. After $48 \mathrm{~h}$, co-cultures that were incubated at 25 and $28^{\circ} \mathrm{C}$ already included high numbers of rounded, detached cells that contained a single, large vacuole filled with motile bacilli. This cytopathic effect was not observed in cultures that were incubated at $35-37^{\circ} \mathrm{C}$, even after prolonged incubation, demonstrating that LLAP $12^{\mathrm{T}}$ maintained stable infection of amoebae at higher temperatures. This ability may contribute to the propensity of man-made water supplies, which are commonly maintained at higher temperatures than that of natural aquatic environments, to serve as sources of Legionella infection. Previous work has demonstrated that the virulence of legionellae increases when they are associated with amoebae (Cirillo et al., 1999); thus, exposure to conditions that are capable of prolonging this association must serve to enhance this pathogenic potential.

\section{Concluding remarks}

Legionellae have been traditionally classified by using polyphasic means, with DNA-DNA hybridization as the
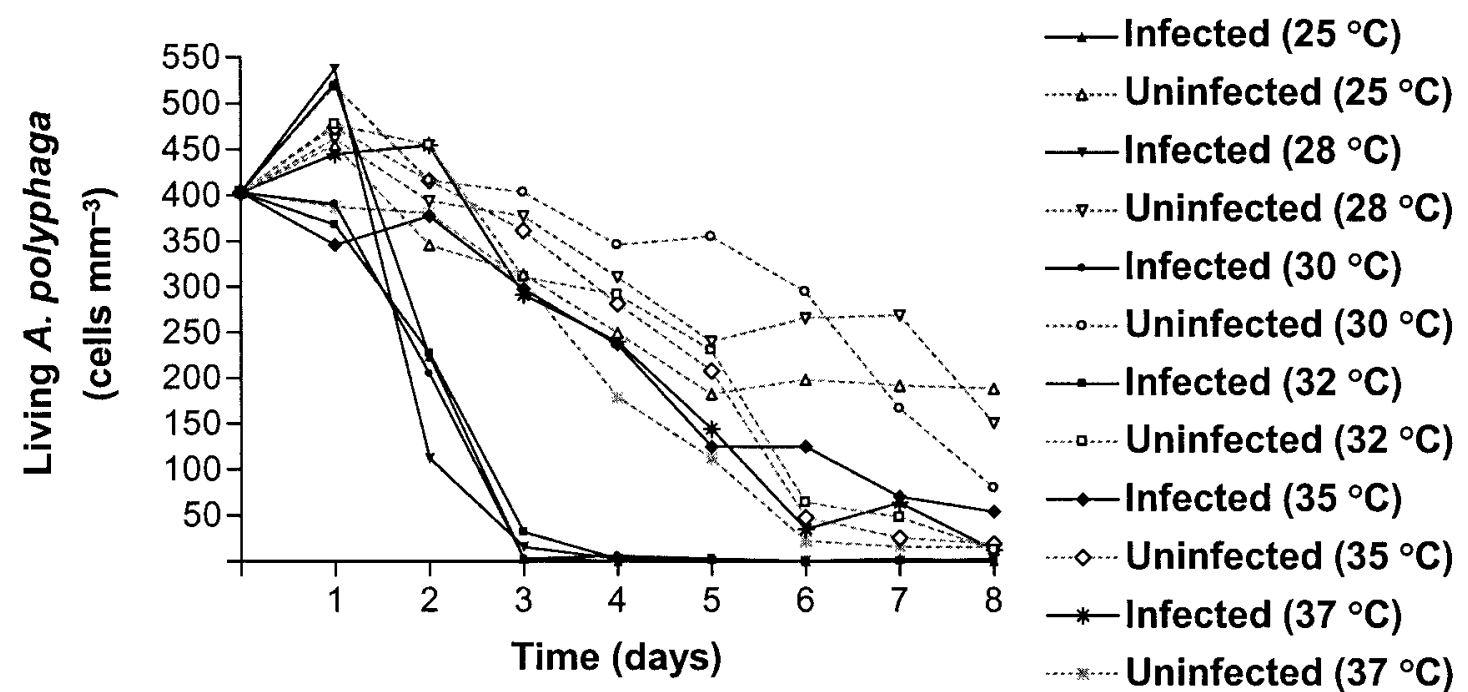

Fig. 3. Effect of temperature on the growth and survival of uninfected (open symbols) and LLAP12 $2^{\top}$-infected (filled symbols) A. polyphaga. 
cornerstone of species delineation. Although a variety of different methods can be utilized in the characterization of intra-amoebal organisms (as demonstrated in this study), the application of techniques that require large amounts of DNA, such as DNA-DNA hybrization, is not feasible. However, it seems foolhardy to exclude organisms such as LLAP $12^{\mathrm{T}}$ from classification, or to even deny them full taxonomic status, merely because of a lack of DNA-DNA hybridization data, if a proposal is well-supported by data derived from other assessments. Clearly, for some intracellular organisms that cannot be sustained in in vitro cultures of eukaryotic cells, concerns could be raised about the uniqueness of isolates, but in the case of LLAP $12^{\mathrm{T}}$ and other LLAPs that have been subjected to limiting dilution and multiple passage, such concerns recede. Furthermore, cultures of LLAP12 ${ }^{\mathrm{T}}$ have been accepted for deposition in culture collections (World Health Organization Collaborative Center for Rickettsial Reference and Research, Marseilles, France, and the American Type Culture Collection, Manassas, VA, USA), thereby providing reference strains for future studies. We propose the name Legionella drancourtii sp. nov. for this organism, in honour of Michel Drancourt, a prolific French microbiologist who has contributed significantly to our understanding of the diversity and nature of intracellular bacteria of medical importance.

\section{Description of Legionella drancourtii sp. nov.}

Legionella drancourtii (dran.cour'ti.i. N.L. gen. n. drancourtii in honour of Michel Drancourt for his work on intracellular micro-organisms, especially Rickettsiae).

Gram-negative. Does not grow on axenic media. Exists naturally as a strictly intracellular parasite of free-living amoebae. Cytopathic effect on A. polyphaga is restricted to co-cultures that are incubated at lower temperatures (25$32{ }^{\circ} \mathrm{C}$ ). May be observed as motile rods within vacuoles of amoebae. DNA G $+\mathrm{C}$ content is $39 \cdot 4 \mathrm{~mol} \%$.

The type strain is $\operatorname{LLAP} 12^{\mathrm{T}}=$ ATCC $50991^{\mathrm{T}}$.

\section{Acknowledgements}

We would like to thank Lina Barrassi for technical help.

\section{References}

Adeleke, A., Pruckler, J., Benson, R., Rowbotham, T., Halablab, M. \& Fields, B. (1996). Legionella-like amebal pathogens - phylogenetic status and possible role in respiratory disease. Emerg Infect Dis 2, 225-230.

Adeleke, A. A., Fields, B. S., Benson, R. F. \& 8 other authors (2001). Legionella drozanskii sp. nov., Legionella rowbothamii sp. nov. and
Legionella fallonii sp. nov.: three unusual new Legionella species. Int J Syst Evol Microbiol 51, 1151-1160.

Ash, C., Farrow, J. A. E., Dorsch, M., Stackebrandt, E. \& Collins, M. D. (1991). Comparative analysis of Bacillus anthracis, Bacillus cereus, and related species on the basis of reverse transcriptase sequencing of 16 S rRNA. Int J Syst Bacteriol 41, 343-346.

Barbaree, J. M., Fields, B. S., Feeley, J. C., Gorman, G. W. \& Martin, W. T. (1986). Isolation of protozoa from water associated with a legionellosis outbreak and demonstration of intracellular multiplication of Legionella pneumophila. Appl Environ Microbiol 51, 422-424.

Barker, J. \& Brown, M. R. (1994). Trojan horses of the microbial world: protozoa and the survival of bacterial pathogens in the environment. Microbiology 140, 1253-1259.

Birtles, R. J., Rowbotham, T. J., Raoult, D. \& Harrison, T. G. (1996). Phylogenetic diversity of intra-amoebal legionellae as revealed by $16 \mathrm{~S}$ rRNA gene sequence comparison. Microbiology 142, 3525-3530.

Cirillo, J. D., Cirillo, S. L. G., Yan, L., Bermudez, L. E., Falkow, S. \& Tompkins, L. S. (1999). Intracellular growth in Acanthamoeba castellanii affects monocyte entry mechanisms and enhances virulence of Legionella pneumophila. Infect Immun 67, 4427-4434.

Fallon, R. J. \& Rowbotham, T. J. (1990). Microbiological investigations into an outbreak of Pontiac fever due to Legionella micdadei associated with use of a whirlpool. J Clin Pathol 43, 479-483.

Fox, G. E., Wisotzkey, J. D. \& Jurtshuk, P. J., Jr (1992). How close is close: $16 \mathrm{~S}$ rRNA sequence identity may not be sufficient to guarantee species identity. Int J Syst Bacteriol 42, 166-170.

Fry, N. K., Rowbotham, T. J., Saunders, N. A. \& Embley, T. M. (1991). Direct amplification and sequencing of the $16 \mathrm{~S}$ ribosomal DNA of an intracellular Legionella species recovered by amoebal enrichment from the sputum of a patient with pneumonia. FEMS Microbiol Lett 67, 165-168.

Harrison, T. G. \& Taylor, A. G. (1988). Identification of legionellae by serological methods. In A Laboratory Manual for Legionella, pp. 57-68. Edited by T. G. Harrison \& A. G. Taylor. London: Wiley. Horn, M., Harzenetter, M. D., Linner, T., Schmid, E. N., Müller, K.-D., Michel, R. \& Wagner, M. (2001). Members of the CytophagaFlavobacterium-Bacteroides phylum as intracellular bacteria of acanthamoebae: proposal of 'Candidatus Amoebophilus asiaticus'. Environ Microbiol 3, 440-449.

La Scola, B., Fenollar, F., Fournier, P.-E., Altwegg, M., Mallet, M.-N. \& Raoult, D. (2001). Description of Tropheryma whipplei gen. nov., sp. nov., the Whipple's disease bacillus. Int J Syst Evol Microbiol 51, 1471-1479.

Ratcliff, R. M., Lanser, J. A., Manning, P. A. \& Heuzenroeder, M. W. (1998). Sequence-based classification scheme for the genus Legionella targeting the mip gene. J Clin Microbiol 36, 1560-1567.

Rowbotham, T. J. (1980). Preliminary report on the pathogenicity of Legionella pneumophila for freshwater and soil amoebae. J Clin Pathol 33, 1179-1183.

Rowbotham, T. J. (1983). Isolation of Legionella pneumophila from clinical specimens via amoebae, and the interaction of those and other isolates with amoebae. J Clin Pathol 36, 978-986.

Stackebrandt, E. \& Goebel, B. M. (1994). Taxonomic note: a place for DNA-DNA reassociation and 16S rRNA sequence analysis in the present species definition in bacteriology. Int $J$ Syst Bacteriol 44, 846-849. 